\title{
Gene Expression in Rat Supraspinatus Tendon Recovers From Overuse With Rest
}

\author{
Scott A. Jelinsky PhD, Spencer P. Lake BS, \\ Joanne M. Archambault PhD, Louis J. Soslowsky PhD
}

Published online: 6 May 2008

(C) The Author(s) 2008

\begin{abstract}
Rest is a common treatment for overuse injuries, but its effectiveness on gene expression has not been systematically evaluated under controlled experimental conditions. We asked whether genes regulated in the supraspinatus tendon as a result of overuse would return to normal levels after 2 or 4 weeks of rest. We used a rat model of tendon overuse that generates reproducible changes in the histology, geometry, gene expression, and mechanical properties consistent with an overuse injury. Animals were subjected to the overuse protocol for 2 or 4 weeks followed by either 2 or 4 weeks of rest. Microarray analysis was used to measure global changes in gene expression after the overuse plus rest protocol. Genes upregulated as a result of the overuse returned to near normal levels after rest in most animals. The biochemical composition of the tendon was similar to normal after the imposed rest period, except for slightly lower collagen content. These results suggest as little as 2 weeks of rest is often sufficient to recover from the molecular and biochemical effects of 2 and 4 weeks of overuse in this rat model.
\end{abstract}

One or more of the authors (SPL, LJS) have received funding from the National Institutes of Health, Wyeth Research, and the National Science Foundation. Two of the authors (SAJ, JMA) are employed by Wyeth Research.

Each author certifies that his or her institution has approved the animal protocol for this investigation and that all investigations were conducted in conformity with ethical principles of research.

S. A. Jelinsky ( $ه)$, J. M. Archambault

Wyeth Discovery Research, 87 Cambridge Park Drive,

Cambridge, MA 02140, USA

e-mail: sjelinsky@wyeth.com

S. P. Lake, L. J. Soslowsky

McKay Orthopedic Research Laboratory, University of

Pennsylvania, Philadelphia, PA, USA

\section{Introduction}

Rotator cuff tendinopathy is a common condition that is increased in certain athletic and occupational environments. Current treatment for a patient with this type of injury begins with rest or activity modification and often includes a course of nonsteroidal antiinflammatory drugs $[4,5,7,9,11,14]$. Rest is believed to allow the tendon to repair itself, however, the effect of rest on an overused tendon has not yet been examined experimentally.

Animal models provide an opportunity to induce injury in a controlled manner and evaluate the effect of rest over time. One rat model of tendon overuse generates reproducible changes in the histology, geometry, and mechanical properties of the supraspinatus tendon consistent with an overuse injury clinically by 4 weeks of exercise $[15,16]$. Specifically, tendon cross-sectional area increases with overuse and modulus and maximum stress decrease relative to control. Structural and cellular changes include increased collagen fiber disorganization, hypercellularity and a change in cell morphology from elongated spindle-shaped cells to more round, plump cells. Recent gene expression profiling analysis also indicates this overuse protocol increases the expression of cartilagespecific genes and reduces the expression of tendonspecific genes in the supraspinatus tendon in a systematic manner. These changes suggest the supraspinatus tendon becomes more like cartilage and less like tendon as a result of overuse [1].

We asked whether genes regulated in the supraspinatus tendon as a result of overuse would return to normal levels after 2 or 4 weeks of rest. At the same time we asked whether biochemical changes were present in the supraspinatus tendon following overuse then rest protocol. 


\section{Materials and Methods}

We subjected rats to a supraspinatus tendon overuse/rest protocol. The overuse protocol consisted of downhill running at $17 \mathrm{~m} / \mathrm{min}$ for 1 hour per day 5 days per week [1,2]. We subjected the animals to either 2 weeks or 4 weeks of running followed by either 2 weeks or 4 weeks of rest as outlined in Fig. 1. We used genomic DNA microarray analysis to simultaneously measure the majority of rat transcripts. We also measured biochemical changes including water, GAG, DNA and collagen content.

Forty-eight male Sprague-Dawley rats $(400-450 \mathrm{~g})$ were subjected to a supraspinatus tendon overuse/rest protocol. Rest consisted of cage activity only after the end of the overuse protocol. We used three experimental groups consisting of 2 weeks of running followed by 2 weeks of rest $(n=8), 4$ weeks of running followed by 2 weeks of rest $(\mathrm{n}=8)$, and 4 weeks of running followed by 4 weeks of rest $(\mathrm{n}=8)$ (Groups 5, 7, 9, respectively) (Fig. 1). Three nonrunning control groups consisting of eight animals each were included so each group had an appropriate time-matched control (Groups 6, 8, 10) (Fig. 1). Seven of eight animals from each of the three run plus rest groups completed the entire protocol. Although sample size for this study was defined based on experimental, rather than statistical, considerations we did run a post hoc power analysis. Of the 196 genes monitored, 139 were expressed above background (set at 50 signal units) and had a median variance of 0.25 on the $\log -2$ scale. A power test using this variance estimate indicates that a two-sample t-test comparison of control animals $(n=8)$ versus treated (overuse plus rest) animals $(\mathrm{n}=5)$, has $69 \%$ power to detect a fold change of 1.7 at a significance level of 0.05 .

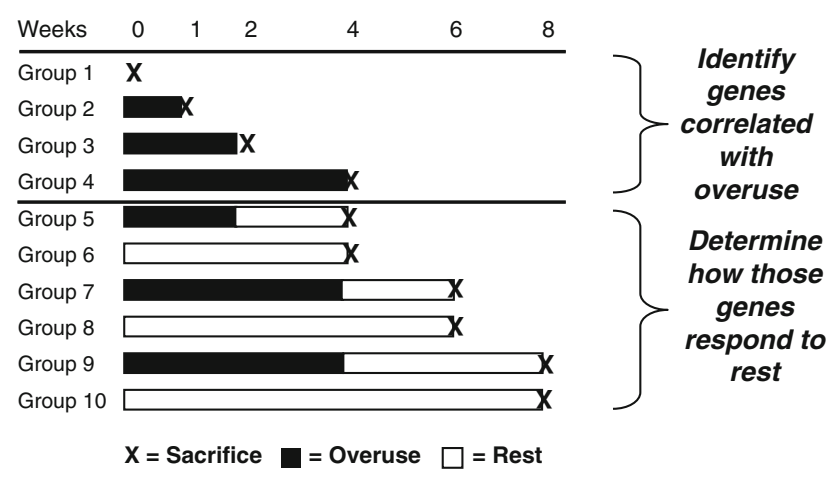

Fig. 1 Experimental design for our earlier [1] and current studies. The earlier study consisted of overuse protocol for 1,2 , or 4 weeks. The current study consisted of overuse followed by rest (normal cage activity). In the current study, three cohorts of overuse followed by rest were evaluated, including 2 -week run followed by 2 -week rest, 4 -week run followed by 2 -week rest, and 4 -week run followed by 4-week rest. Also in the current study, three cohorts of age-matched cage activity controls were included.
We obtained prior institutional Animal Care and Use Committee approval. Animals were housed in pairs with unrestricted cage activity and were given food and water ad libitum. After protocol completion, rats were sacrificed with an overdose of carbon dioxide. At necropsy, the supraspinatus tendon from each shoulder was removed and snapfrozen in liquid nitrogen. One tendon from each rat was freeze-fractured and extracted using TRIzol reagent (Invitrogen, Carlsbad, CA). RNA was isolated from the aqueous phase of the extract using an RNeasy kit (QIAGEN, Valencia, CA) as described previously [1]. RNA concentrations were determined using a spectrophotometer and the RNA stored at $-80^{\circ} \mathrm{C}$ until needed. Two to $5 \mu \mathrm{g}$ of total RNA was used to generate biotin-labeled cRNA using an oligo T7 primer in a reverse transcription reaction followed by an in vitro transcription reaction with biotin-labeled UTP and CTP. Ten micrograms of cRNA were fragmented and hybridized to a rat genome array (RAE230 2.0; Affymetrix, Santa Clara, CA), which monitors over 30,000 transcripts from over 28,000 unique genes. Hybridized arrays were stained and scanned according to the manufacturer's protocols (Fluidics Station 450 and Affymetrix scanner 3000; Affymetrix). We visually inspected all array images for defects and quality. Arrays with excessive background, low signal intensity, major defects within the array, or poor-quality control metrics (measured by the $5^{\prime}-3^{\prime}$ ratio of GAPDH and $\beta$-actin) were eliminated from further analysis. Furthermore, in vitro synthesized transcripts of 11 bacterial transcripts were included in each hybridization reaction. The abundance of these transcripts ranged from 1:300000 (3 ppm) to 1:1000 (1000 ppm) stated in terms of the number of control transcripts per total transcripts [6]. These bacterial transcripts were utilized for the derivation of gene chip profile quality control (QC) metrics as well determination of the limit of sensitivity for each individual array. Four samples passed QC for the 2-week run plus 2-week rest (Group 5), five samples passed QC for the 4-week run plus 2-week rest (Group 7), and seven samples passed QC for the 4-week run versus 4-week rest (Group 9). In the control samples, eight, eight, and seven samples passed QC for the 4-, 6-, and 8-week rest cohorts, respectively.

We determined signal values using the Gene Chip Operating System (GCOS 1.0; Affymetrix). For each array, all probe sets were normalized to a mean signal intensity value of 100 . The default GCOS statistical values were used for all analysis. Signal values and absolute detection calls were imported into Expressionist (GeneData, Basel, Switzerland) for analysis.

We considered a gene detectable if the mean expression in any sample was greater than 50 signal units and the percentage of samples with a Present $(\mathrm{P})$ call as determined by GCOS default settings was greater than or equal to $66 \%$. Normalized signal values were transformed to the log base 
10. To account for possible interexperiment biases between the current study and our previous study [1], we used the nonrunning experimental groups that were common between studies (Groups 1, 6, 8, 10) (Fig. 1) as reference groups for their respective experiments. For both studies, expression of each transcript in each sample was divided by the geometric mean expression of the same transcript in the reference nonrunning groups in that experiment. In this manner, all expression values could be reported as fold changes relative to the nonrunning control group in the same experiment.

The following method was performed to identify whether the expression of genes was affected by the overuse/rest protocol and was limited to the 196 genes previously identified as regulated by overuse [1]. The three nonrunning control groups (Groups 6, 8, 10) (Fig. 1) were combined into a single group since only a small fraction of the genes (13) had any differences (ANOVA $\mathrm{p}<0.01$ ) in mRNA expression). Individual Welch's t-test on logtransformed data was performed on each mRNA measurement from each of the three run plus rest groups (Groups 5, 7,9) (Fig. 1) compared to the combined control group (Groups 6, 8, 10) (Fig. 1). Fold change values were calculated on the log transformed normalized data.

We used the contralateral supraspinatus tendon from each rat for biochemical analysis. Water content was determined by weighing the tissue before and after being lyophilized. The dried tendons were digested overnight with proteinase $\mathrm{K}$ and then centrifuged to remove insoluble components. We used a small aliquot of the digestate to determine the glycosaminoglycan (GAG) content with a dimethylmethylene blue colorimetric assay [10]. The DNA content was determined in the digestate using a fluorescent dye (PicoGreen; Molecular Probes, Carlsbad, CA). The remainder of the digestate was hydrolyzed overnight with $6 \mathrm{~N} \mathrm{HCl}$ and then assayed for hydroxyproline content with Erlich's reagent [12]. The hydroxyproline level in the tendon reflects its collagen content, the DNA content is a measure of its cellularity, and the GAG content is a measure of the proteoglycan content. Results were expressed per milligram of dry weight for each tendon.

For the biochemical analysis, the three control groups were combined (Groups $6,8,10$ ) and compared with the tendons from the three run plus rest groups using analysis of variance. If a difference $(p<0.05)$ between groups was detected for one of the biochemical variables (water, GAG, DNA or collagen content), then post hoc contrasts were performed using the Scheffe test (JMP 5, SAS, Cary, NC).

\section{Results}

An imposed rest period following overuse protocol allowed mRNA expression levels to return to near normal levels.
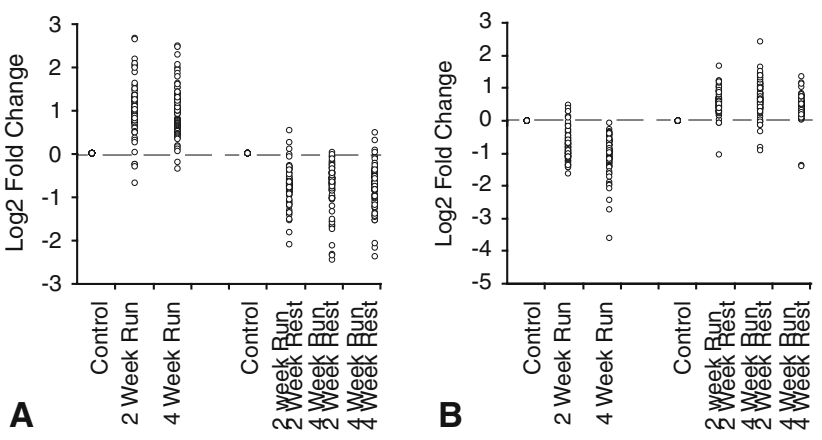

Fig. 2A-B Mean expression of 196 genes regulated by overuse is shown [1]. The majority of genes regulated by overuse return to near control levels after the run/rest protocol. Shown is the fold change relative to the nonrunning (Group 1) from that earlier study [1] or the fold change relative to average expression of combined age-matched control (Groups 6, 8, 10) from this study for (A) genes induced after the running protocol or $(\mathbf{B})$ repressed after the running protocol.

The expression of the 196 genes was affected by rest. Eighty-six genes were upregulated following the overuse protocol of 2 and 4 weeks and their expression level returned to near baseline levels after as little as 2 weeks of rest (Fig. 2A). Of the 86 overuse-induced transcripts, only six (Cry1, Fst, Mybph, Sln, and two uncharacterized transcripts) had an increase ( $p<0.05$, fold change less than 1.7) in expression following the overuse/rest protocol at any time point, whereas the expression of 22 (including Calcr1, Cav, Clic4, Epas1, Igf1, Mob1, Tm9sf3, Mcpt1, Rab5b, Tgfb3, Sep2, Sec61a1, Thy1, and Timp3) overuse-induced transcripts had a decrease $(\mathrm{p}<0.05$, fold change less than -1.7 ) in expression following the overuse/rest protocol. Similarly, 110 genes were down regulated following the overuse protocol and their expression level returned to near baseline levels with rest (Fig. 2B). Of the 110 overuserepressed transcripts, the expression of only nine genes (including Aqp4, Myl3, Pkia, Myoz2, and Cabc1) differed $(\mathrm{p}<0.05$, fold change less than -1.7$)$ at any of the time points following the overuse/rest protocol, whereas expression of five (including Dgat2, Ephx2, and Nr1d1) of these genes increased following the overuse/rest protocol. The expression level of cartilage- and tendon-expressed genes [1] also returned to baseline levels following overuse/ rest protocol. The expression patterns of two cartilageexpressed genes (Col2 and Agc1) (Fig. 3) and two tendonspecific genes (Col1a1 and Igf1) (Fig. 4) returned to near control levels following the run plus rest protocol.

The biochemical composition of the tendons from the animals subjected to the overuse plus rest protocol generally supports the findings of the molecular analysis; the biochemical composition was generally similar to that at baseline. Specifically the water, DNA, and glycosaminoglycan content of the tendons of animals in Groups 5, 7, and 9 were similar to those in tendons in age-matched control animals (Fig. 5). 


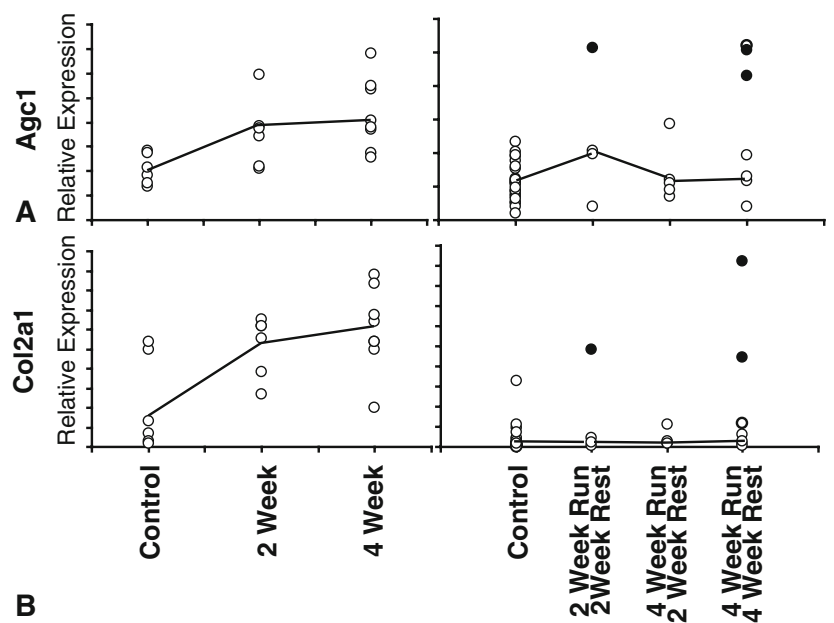

Fig. 3A-B The mRNA levels of cartilage selective genes aggrecan 1 (Agc1) and collagen, type2, alpha 1 (Col2a1) return to normal levels after the run/rest protocol. The relative mRNA levels for (A) Agc1 and (B) Col2a1 are shown for each individual sample. Highlighted are animals that failed to recover after rest. The data were $\mathrm{z}$ scored normalized and expressed as relative ratio to control levels within the earlier [1] and current studies.

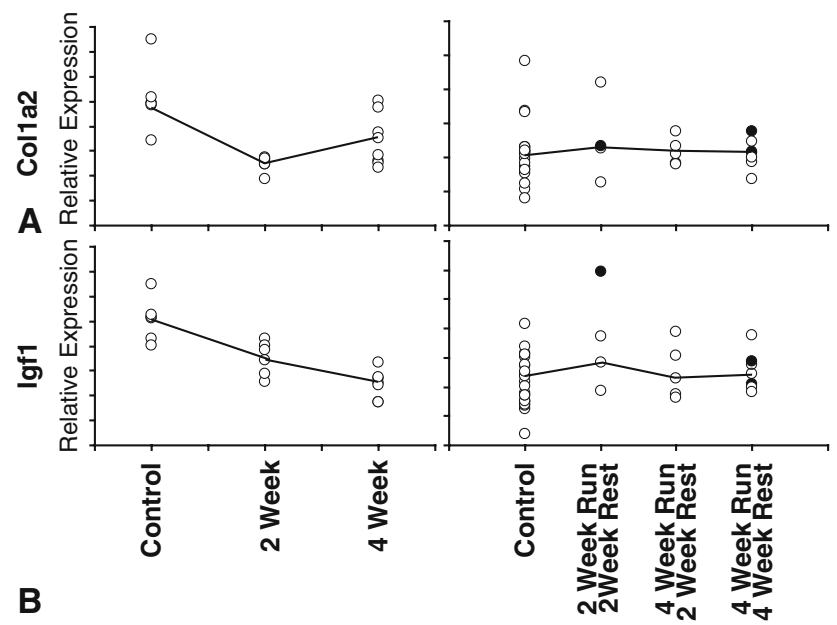

Fig. 4A-B The mRNA levels of tendon selective genes collagen, type 1, alpha 2 (Col1a2), and insulin growth factor 1 (Igf1) return to normal levels after the run/rest protocol. The relative mRNA levels for (A) Col1a2 and (B) Igf1 are shown for each individual sample. Highlighted are animals that failed to recover after rest. The data were $\mathrm{z}$ scores normalized and expressed as relative ratio to control levels within the earlier [1] and current studies.

However, the collagen content of the animals in the run plus rest groups as a whole was lower $(\mathrm{p}=0.039)$ than that in the tendons of age-matched control animals.

\section{Discussion}

While current treatment for patients with rotator cuff tendinopathy often includes behavior modification and rest,

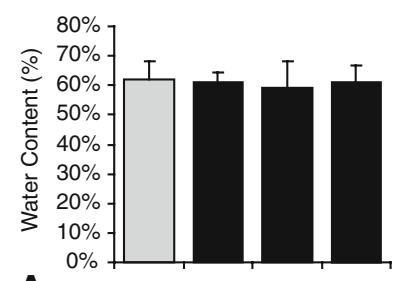

A
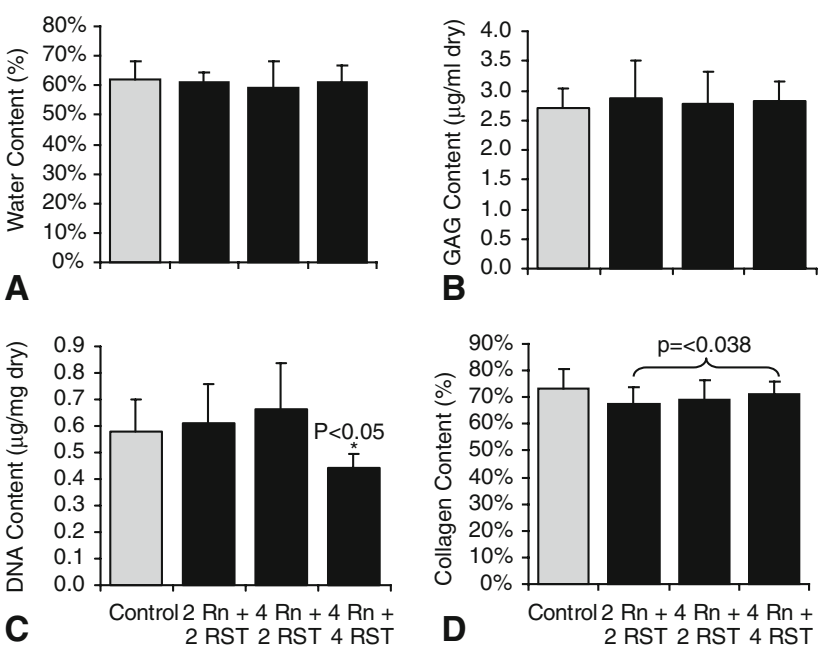

Fig. 5A-D Biochemical composition of rat supraspinatus tendon. The (A) average water, (B) glycosaminoglycan, (C) DNA, and (D) collagen content for each group are shown.

the changes induced by rest have not been completely understood. We asked whether genes regulated in the supraspinatus tendon as a result of overuse would return to normal levels after 2 or 4 weeks of rest and whether biochemical changes persisted in the supraspinatus tendon following overuse then rest protocol.

The design of this study has limitations that affect the interpretation of the results. First, it is unknown to what degree this rat model replicates the human in terms of gene expression and timing of expression with injury and rest. Second, we cannot show that gene expression was altered from normal at the end of the overuse period, before the rest began. We only know that in an earlier experiment [1], the expression of certain genes was considerably altered by the same overuse stimulus. One other report suggested overuse induces substantial changes in gene expression in this animal model [8]. Third, the biochemical composition of the tendons was not measured in our earlier study [1], so the current biochemical results cannot be compared with overuse-only animals. However, GAG content is increased in this model as a function of overuse [13]. Fourth, although our earlier [1] and current study have identified many genes that are highly regulated with overuse and rest, the amounts of corresponding proteins present for these genes is unknown because protein levels were not measured. Because protein translation, activation, and degradation are regulated independently of gene expression, direct correlations between gene regulation and amount of active protein cannot be assumed. In addition, the function and the mechanical properties of tendon have not been evaluated to see if they have returned to normal with rest. Finally, a short rest period was seen to restore normal gene expression for most animals that had been subjected to 2 or 4 weeks of overuse. It is unknown 
whether a similar rest period would also cause recovery for animals subjected to a longer period of overuse or whether recovery would be possible at all for these animals even with a long rest period. Further study will need to evaluate the effect of rest on longer-term overuse in this model.

Three of the 24 animals in the overuse plus rest protocol had gene expression levels for the cartilage-specific genes comparable to animals in the overuse only protocol [1], suggesting these animals may have had a different response to rest. Two of the seven animals from Group 9 (Fig. 1) had higher expression of the cartilage-specific genes although expression of other genes returned to control levels. For 13 of the 17 cartilage-specific genes, these two animals averaged at least a 1.7-fold higher expression compared with the remaining Group 9 animals and the expression level of $\mathrm{Col} 2$ and Agc1 had a 17-fold and fourfold higher expression in these animals, respectively. In contrast, only six of the remaining 178 genes had a greater than 1.7-fold change in expression in these two animals. Furthermore, principal component analysis suggested these animals had a distinct expression pattern compared with the rest of the Group 9 animals (data not shown). Similarly, one animal from Group 5 (Fig. 1) appeared to have higher expression of the cartilage-specific genes. For eight of the 17 cartilage-specific genes, this animal averaged at least a 1.8-fold higher expression compared with the remaining Group 5 animals. As a result of the particularly different nature of these data, these animals were not included in the overall analysis, although results from these animals are described for completeness.

We earlier reported that genes that are highly expressed in cartilage were upregulated, and conversely, genes that are highly expressed in tendon were downregulated in the supraspinatus tendon as a result of 2 and 4 weeks of the overuse protocol [1]. The inclusion of a rest period after the overuse protocol in the current study has resulted in the expression of these genes returning to normal, nonrunning levels in most animals. This result suggests the removal of the overuse ( 2 or 4 weeks) stimulus allowed gene expression in the tendon tissue to revert to normal with as little as 2 weeks of rest. However, the composition and function of the tissue may still be altered from normal as a result of the initial overuse stimulus. For example, although the DNA and GAG content returned to normal levels after rest, the collagen content of the tendons had not returned to normal levels despite the imposed rest period.

Our findings suggest there is a scientific basis for the use of rest as at least one component of treatment for tendinopathy. It is well-known cells, including those found in tendon, are sensitive to mechanical stimuli $[2,3,17,18]$. The removal of a stimulus can be as important as the explicit introduction or increase of a stimulus. One would expect cells would reach a new set point. In this study, the removal of the overuse stimulus was a signal to the cells in the tendon to adapt to this new lower level of stimulus. However, the tendons in three of the animals in this study did not adapt to the remainder in the expected manner. It is possible the cells in these tendons were more damaged from the overuse stimulus than in the others such that they were unable to respond to the lowered mechanical demands after removal of the overuse. This highlights the potential impact of genetic factors, biologic variation, and multifactorial considerations in the response of tendons to overuse and rest.

Although rest has often been prescribed clinically for patients with overuse tendon injuries, the biological effects of rest on tendinopathy are not well known. Using a rat model of repetitive motion overuse, we demonstrated 2 weeks of rest is sufficient to restore normal gene expression in most animals subjected to 2 or 4 weeks of treadmill running. In addition, although collagen content was slightly decreased in run plus rest groups compared with controls, the DNA, GAG, and water content were not different from controls for these groups. Therefore, most measures of biochemical content analyzed in this study also demonstrated a recovery to normal levels after rest. Although this study indicates beneficial effects with rest, future work is needed to evaluate other measures of tendon properties (e.g., protein levels, mechanical properties) to fully understand the effect of rest on tendon injury and repair.

Acknowledgments We thank Andrew Hill for providing advice on the statistical analysis and Ewa Wilson for generating the microarray data.

Open Access This article is distributed under the terms of the Creative Commons Attribution Noncommercial License which permits any noncommercial use, distribution, and reproduction in any medium, provided the original author(s) and source are credited.

\section{References}

1. Archambault JM, Jelinsky SA, Lake SP, Hill AA, Glaser DL, Soslowsky LJ. Rat supraspinatus tendon expresses cartilage markers with overuse. J Orthop Res. 2007;25:617-624.

2. Arnoczky SP, Tian T, Lavagnino M, Gardner K. Ex vivo static tensile loading inhibits MMP-1 expression in rat tail tendon cells through a cytoskeletally based mechanotransduction mechanism. J Orthop Res. 2004;22:328-333.

3. Banes AJ, Horesovsky G, Larson C, Tsuzaki M, Judex S, Archambault J, Zernicke R, Herzog W, Kelley S, Miller L. Mechanical load stimulates expression of novel genes in vivo and in vitro in avian flexor tendon cells. Osteoarthritis Cartilage. 1999;7:141-153.

4. Blair B, Rokito AS, Cuomo F, Jarolem K, Zuckerman JD. Efficacy of injections of corticosteroids for subacromial impingement syndrome. J Bone Joint Surg Am. 1996;78:1685-1689.

5. Fredberg U. Local corticosteroid injection in sport: review of literature and guidelines for treatment. Scand J Med Sci Sports. 1997;7:131-139.

6. Hill AA, Brown EL, Whitley MZ, Tucker-Kellogg G, Hunter CP, Slonim DK. Evaluation of normalization procedures for 
oligonucleotide array data based on spiked cRNA controls. Genome Biol. 2001;2:1-13.

7. McConville OR, Iannotti JP. Partial-thickness tears of the rotator cuff: evaluation and management. J Am Acad Orthop Surg. 1999;7:32-43.

8. Molloy TJ, Kemp MW, Wang Y, Murrell GA. Microarray analysis of the tendinopathic rat supraspinatus tendon: glutamate signaling and its potential role in tendon degeneration. $J$ Appl Physiol. 2006;101:1702-1709.

9. Morrison DS, Frogameni AD, Woodworth P. Non-operative treatment of subacromial impingement syndrome. J Bone Joint Surg Am. 1997;79:732-737.

10. Muller G, Hanschke M. Quantitative and qualitative analyses of proteoglycans in cartilage extracts by precipitation with 1,9-dimethylmethylene blue. Connect Tissue Res. 1996;33:243248.

11. Mulligan E. Conservative management of shoulder impingement syndrome. J Athl Train. 1988;23:348-353.

12. Reddy GK, Enwemeka CS. A simplified method for the analysis of hydroxyproline in biological tissues. Clin Biochem. 1996;29: 225-229.
13. Scott A, Cook JL, Hart DA, Walker DC, Duronio V, Khan KM. Tenocyte responses to mechanical loading in vivo: a role for local insulin-like growth factor 1 signaling in early tendinosis in rats. Arthritis Rheum. 2007;56:871-881.

14. Sommerich CM, McGlothlin JD, Marras WS. Occupational risk factors associated with soft tissue disorders of the shoulder: a review of recent investigations in the literature. Ergonomics. 1993;36:697-717.

15. Soslowsky LJ, Carpenter JE, DeBano CM, Banerji I, Moalli MR. Development and use of an animal model for investigations on rotator cuff disease. J Shoulder Elbow Surg. 1996;5:383-392.

16. Soslowsky LJ, Thomopoulos S, Tun S, Flanagan CL, Keefer CC, Mastaw J, Carpenter JE. Neer Award 1999. Overuse activity injures the supraspinatus tendon in an animal model: a histologic and biomechanical study. J Shoulder Elbow Surg. 2000;9:79-84.

17. Wall ME, Banes AJ. Early responses to mechanical load in tendon: role for calcium signaling, gap junctions and intercellular communication. J Musculoskelet Neuronal Interact. 2005;5: $70-84$.

18. Wang JH. Mechanobiology of tendon. J Biomech. 2006;39:15631582 . 